\title{
Unveiling Molecular Changes in Water by Small Luminescent Nanoparticles
}

Lucía Labrador-Páez, Dragana J. Jovanovic', Manuel I. Marqués, Krisjanis Smits, Slobodan D. Dolic', Francisco Jaque, Harry Eugene Stanley, Miroslav D. Dramic'anin, José García-Solé, Patricia Haro-González, and Daniel Jaque*

\begin{abstract}
Nowadays a large variety of applications are based on solid nanoparticles dispersed in liquids-so called nanofluids. The interaction between the fluid and the nanoparticles plays a decisive role in the physical properties of the nanofluid. A novel approach based on the nonradiative energy transfer between two small luminescent nanocrystals $\left(\mathrm{GdVO} 4: \mathrm{Nd}^{3+}\right.$ and $\mathrm{GdVO}_{4}: \mathrm{Yb}^{3+}$ ) dispersed in water is used in this work to investigate how temperature affects both the processes of interaction between nanoparticles and the effect of the fluid on the nanoparticles. From a systematic analysis of the effect of temperature on the GdVO4: $\mathrm{Nd}^{3+} \rightarrow \mathrm{GdVO} 4: \mathrm{Yb}^{3+}$ interparticle energy transfer, it can be concluded that a dramatic increase in the energy transfer efficiency occurs for temperatures above $45^{\circ} \mathrm{C}$. This change is properly explained by taking into account a crossover existing in diverse water properties that occurs at about this temperature. The obtained results allow elucidation on the molecular arrangement of water molecules below and above this crossover temperature. In addition, it is observed that an energy transfer process is produced as a result of interparticle collisions that induce irreversible ion exchange between the interacting nanoparticles.
\end{abstract}

\section{Introduction}

Luminescent nanofluids (LNFs) are colloidal suspensions of luminescent nanoparticles (LNPs) in liquid media. LNFs have recently emerged as versatile and powerful tools capable of providing innovative solutions in a wide range of fields, including analytical chemistry, renewable ener-gies, and biomedicine. $^{[1]}$ From a fundamental point of view, LNFs constitute a novel scenario for the study of the physical mechanisms governing interaction processes between nanoparticles, and the effect of the solvent on the nanoparticles at the nanoscale level by using the luminescence generated 
by LNPs as a tool. For instance, approaches already used to get information on these interaction mechanisms involve the study of the Brownian colloidal nature of LNFs due to the interaction of the molecules of the fluid with the LNPs, ${ }^{[2]}$ radiation trapping between LNPs, ${ }^{[3]}$ the enhancement of the stability of the luminescence of silica nanocrystals, ${ }^{[4]}$ or even efficient nonradiative energy transfer processes between LNPs in close proximity. ${ }^{[5]}$ The physics of LNFs is even more mysterious and challenging when dealing with colloidal small $(\approx 3 \mathrm{~nm})$ luminescent nanoparticles (SLNPs). In such small NP sizes, over $80 \%$ of constituent atoms are located on the surface. Therefore, an enormous fraction of the constituent atoms is in physical contact with the solvent molecules or with other SLNPs. As a consequence, any change in the envi-ronment of an SLNP or even the interparticle interactions affects most of their atoms, and thus has a major impact on their luminescent properties. Thus, we expect that a thorough analysis of the luminescence generated by LNFs containing SLNPs will provide information about both the molecular status of the liquid solvent and the nature and magnitude of interaction events between SLNPs.

The use of LNFs for nanoscale molecular investiga-tions is especially exciting when applied to water-the most common substance on earth and indispensable in life and human development. Although water has been studied for decades and most of its properties are well known, the inter-actions between water molecules are not fully understood. In particular, thermal anomalies in liquid water properties are still a matter of debate. ${ }^{[6]}$ Indeed most water proper-ties have been studied by macroscopic techniques, such as optical and dielectric spectroscopy, and they do not provide details at the molecular level. Questions about the molecular dynamics of water remain unanswered, such as a crossover at around $45{ }^{\circ} \mathrm{C}$ that affects a large variety of water properties and the thermal stability of biological macromolecule as pro-teins. ${ }^{[7]}$ As this anomaly takes place within the physiological temperature range, it is expected to have a great impact in the use of nanofluids for biomedical applications. ${ }^{[8]}$ The use of nanoscopic techniques capable of monitoring dynamic molecular interactions at the molecular scale will enable us to better understand the dynamics of water, the use of LNFs containing SLNPs in particular.

The goal of this work is to understand the crossover in the molecular behavior of water at $\approx 45{ }^{\circ} \mathrm{C}$ by means of inter-particle energy transfer (IPET) between two different kind of SLNPs (donor and acceptor SLNPs), both coexisting in the same LNF. ${ }^{[9]}$ As previously reported by Sarkar et al., ${ }^{[9 \mathrm{~b}]}$ after the optical excitation of donor SLNPs, energy transfer to the acceptor SLNPs may occur via collision-assisted processes caused by the thermal motion of both donor and acceptor SLNPs in the solvent fluid. ${ }^{[2]}$ Thus energy transfer in colli-sion-assisted IPET (CA-IPET) takes place when the donor and acceptor SLNPs collide, such that the superficial lumi-nescent ions of the interacting SLNPs are in a distance range allowing nonradiative energy transfer. CA-IPET efficiency $(\varphi \mathrm{CA}-\mathrm{IPET})$ is strongly correlated with the molecular struc-ture of the solvent and, consequently, temperature-induced changes in the water molecular properties will strongly affect

\section{Results and Discussion}

The used SLNPs consist of GdVO4 small nanocrystals doped with either $\mathrm{Nd}^{3+}$ (donor nanoparticles) or $\mathrm{Yb}^{3+}$ ions (acceptor nanoparticles), hereafter designated SLNPs:Nd and SLNPs:Yb, respectively. Trivalent rare-earth-ion-doped SLNPs are excellent optical probes and display narrow absorption and emission bands. ${ }^{[10]}$ In addition, the energy levels of both $\mathrm{Nd}^{3+}$ and $\mathrm{Yb}^{3+}$ lead to a phonon-assisted spectral overlap between the emission of $\mathrm{Nd}^{3+}$ ions and the absorption of $\mathrm{Yb}^{3+}$ ions, which ensures the possibility of an efficient $\mathrm{Nd}^{3+} \rightarrow \mathrm{Yb}^{3+}$ nonradiative energy transfer when the SLNPs:Nd and SLNPs:Yb are close to each other. ${ }^{[11]}$ Details about synthesis procedure and morphological properties of these SLNPs are supplied in the Experimental Section and in Sections S1 and S2 (Supporting Information). Figure 1a,b shows that the average diameter of both SLNPs:Nd and SLNPs: $Y$ b is $D_{\mathrm{NP}}=2.9 \pm 0.5 \mathrm{~nm}$. Figure 1a shows a transmission electron microscopy (TEM) image revealing that the crystallographic structure is preserved even at small sizes. Figure 1c shows digital pictures of the SLNPs:Nd and SLNPs:Yb LNFs, which were prepared separately in stable colloidal aqueous suspensions. Figure 1e displays a digital picture of LNF containing both SLNPs:Nd and SLNPs:Yb, which was obtained by mixing certain volumes of the original suspensions. Both original and mixed LNFs are colloidal, and even over a period of months do not precipitate. Figure 1d shows a room temperature emission spectra generated by the original (unmixed) LNFs under 808-nm laser excitation, i.e., under a selective excitation of $\mathrm{Nd}^{3+}$ ions (see the absorp-tion of LNFs in Figure S7 in the Supporting Information). As expected, no luminescence is generated by the LNF containing only SLNPs:Yb (see also Figure S8 in the Supporting Information). On the other hand, the emission spec-trum generated by the LNFs containing only SLNPs:Nd in the studied spectral range $(950-1125 \mathrm{~nm})$ is the well-known

${ }^{4} \mathrm{~F}_{3 / 2} \rightarrow{ }^{4} \mathrm{I} 11 / 2$ infrared emission band of $\mathrm{Nd}^{3+}$ ions centered at $\approx 1060 \mathrm{~nm}$. Taking into account the energy level diagrams of $\mathrm{Nd}^{3+}$ and $\mathrm{Yb}^{3+}$ ions (shown schematically in Figure 1c,e), only SLNPs:Nd generate luminescence under 808-nm excitation (which matches the ${ }^{4} \mathrm{I} / 2 \rightarrow{ }^{4} \mathrm{~F}_{5 / 2}$ absorption band of $\mathrm{Nd}^{3+}$ ions). Figure 1f shows the emission spectra (optically excited by $808-\mathrm{nm}$ laser light) obtained at different temperatures when the LNF contains both SLNPs:Nd and SLNPs:Yb. All display the 1060-nm emission band of $\mathrm{Nd}^{3+}$ and the characteristic emission band of $\mathrm{Yb}^{3+}$ centered at $980 \mathrm{~nm} \mathrm{(}{ }^{2} \mathrm{~F}_{5 / 2} \rightarrow{ }^{2} \mathrm{~F} 7 / 2$ transition), revealing that $\mathrm{Nd}^{3+} \rightarrow \mathrm{Yb}^{3+}$ energy transfer takes place, i.e., the IPET process. In this IPET process, SLNPs:Nd are donor units that absorb $808 \mathrm{~nm}$ of excitation energy and par-tially transfer energy to the SLNPs:Yb acceptor units. Note that for close to ambient temperatures $\left(20-30{ }^{\circ} \mathrm{C}\right.$ in Figure 1f) we see only weak traces of $\mathrm{Yb}^{3+}$ ion emission, but when tem-peratures exceed $45{ }^{\circ} \mathrm{C}\left(80{ }^{\circ} \mathrm{C}\right.$ in Figure 1f) we observe a substantial increase in the $\mathrm{Yb}^{3+}$ ions emission and a corre-sponding decrease in the emission of $\mathrm{Nd}^{3+}$ ions, which indi-cates a strong increase in the IPET. The nonradiative nature of this IPET process has been confirmed by the shortening of the emission lifetime of the donor ion $\left(\mathrm{Nd}^{3+}\right)$ in the presence of SLNPs:Yb (see Section S3 in the Supporting Information). 

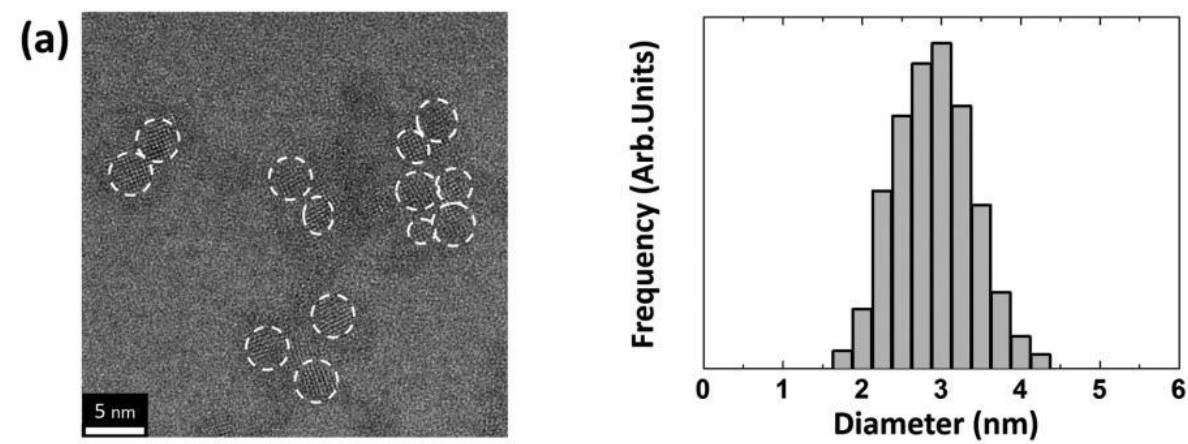

(b)
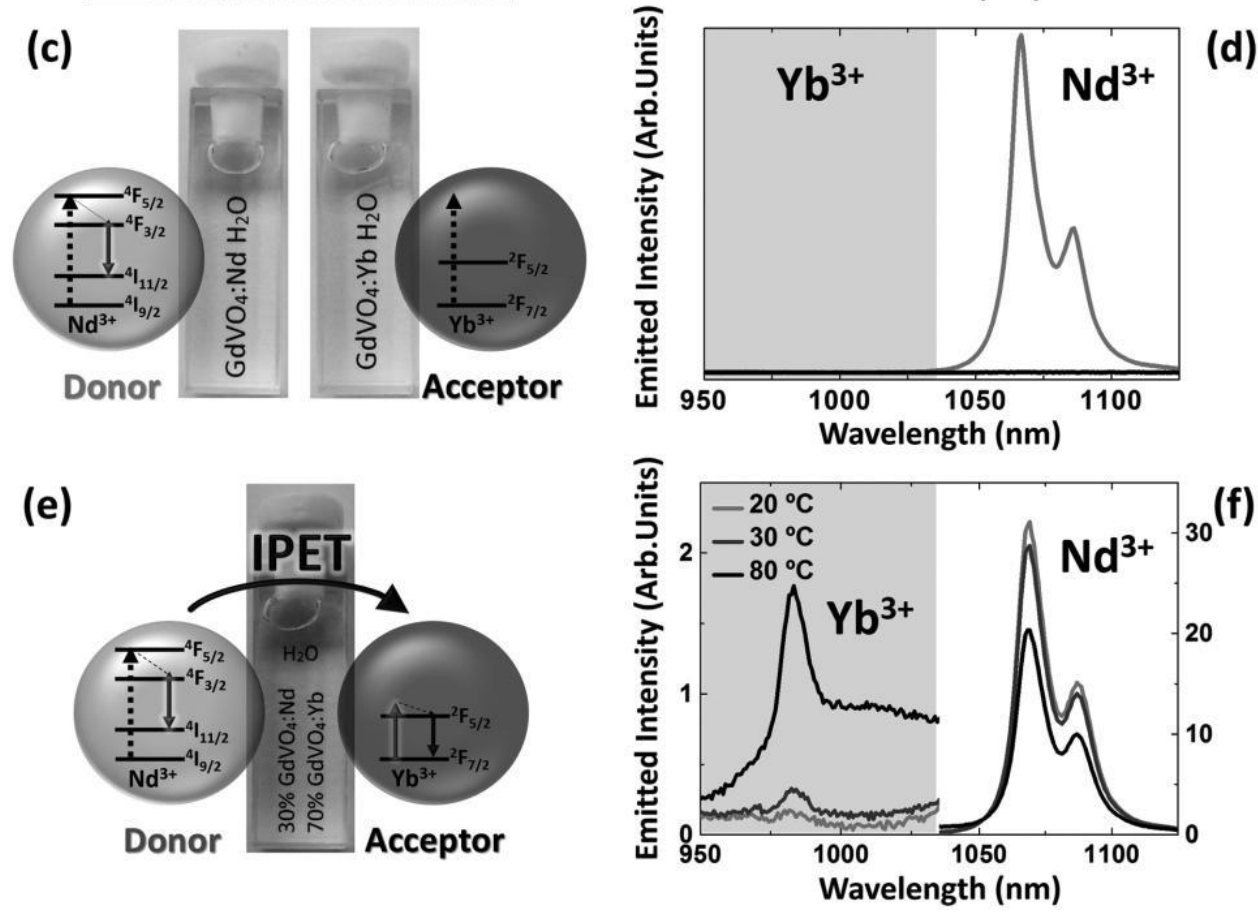

Figure 1. Characterization of the SLNPs and emission of the LNFs. a) TEM image of a mix of SLNP:Nd and SLNP:Yb (dashed circles show the positions of the SLNPs). b) Size distribution obtained from the analysis of TEM images. C) Schematic representation of the energy levels of $\mathrm{Nd}^{3+}$ and $\mathrm{Yb}^{3+}$ ions accounting for the emission (dotted arrow) obtained under selective excitation of $\mathrm{Nd}^{3+}$ ions (808 $\mathrm{nm}$; gray arrow) and optical image of the cuvettes containing the LNFs with either SLNP:Nd or SLNP:Yb. d) Emission spectra of the LNFs shown in (c). e) Schematic representation of the energy levels of $\mathrm{Nd}^{3+}$ and $\mathrm{Yb}^{3+}$ ions accounting for the emissions (gray arrow Nd ${ }^{3+}$ and black arrow $\mathrm{Yb}^{3+}$ ) obtained from the mixed LNF under optical excitation of $\mathrm{Nd}^{3+}$ ions (dotted arrow) and the IPET process (an optical image of the cuvette containing the mixed LNF is also shown). f) Emission spectra of this LNF at three different temperatures.

Thus the data in Figure 1 evidence the presence of IPET in LNFs containing both SLNPs:Nd and SLNPs:Yb, but it does not reveal the responsible mechanism. The litera-ture indicates two possible mechanisms that could explain IPET. The first one is the well-known Dexter-Förster IPET (DF-IPET) that assumes that electric dipole interaction causes noncontact energy transfer between donor and acceptor units. The second one is the CAIPET described above, ${ }^{\left[{ }^{[b]}\right.}$ in which the IPET from donor to acceptor particles occurs when they collide. As explained in Section S4 (Sup-porting Information), we can discriminate between DF-IPET

and CA-IPET by studying the dependence of $\varphi_{\text {CA-IPET on fluid }}$ viscosity $(\eta)$. An increase in $\eta$ reduces the collision rate,

and thus $\varphi$ CA-IPET, for a CA-IPET process. On the other hand, $\eta$ does not affect $\varphi$ IPET for a dominant DF-IPET pro-cess. Figure 2a shows the emission spectra obtained from mixed LNFs of different $\eta$ that preserve the same average interparticle distances. The procedure for controlling the $\eta$ variation is described in the Experimental Section. Figure $2 b$ shows how $\varphi$ CA-IPET decreases as the solvent fluid $\eta$ increases. We also obtain a $\varphi$ CA-IPET $\propto 1 / \eta$ trend, identical to that expected for a pure CA-IPET process (see Section S4 in the Supporting Information). We thus conclude that collisions are the dominant mechanism leading to the interparticle energy transfer in the mixed LNF.

Figure 3a shows the dependence of $\varphi_{\mathrm{CA}}-$ IPET on the LNF temperature in the $20-80{ }^{\circ} \mathrm{C}$ range obtained during a heating and cooling cycle. Two regimes are observed during the heating process. In the $10-40{ }^{\circ} \mathrm{C}$ range, the

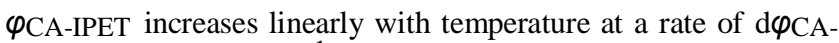
IPET $/ \mathrm{d} T \approx 0.45 \%{ }^{\circ} \mathrm{C}^{-1}$. In the $60-80{ }^{\circ} \mathrm{C}$ range, $\varphi$ CA-IPET increases linearly with temperature but with a much larger rate of $\mathrm{d} \varphi \mathrm{CA}$-IPET $/ \mathrm{d} T \approx 3.2 \%{ }^{\circ} \mathrm{C}^{-1}$. Note that these two regimes cannot be correlated to morphological changes affecting the 

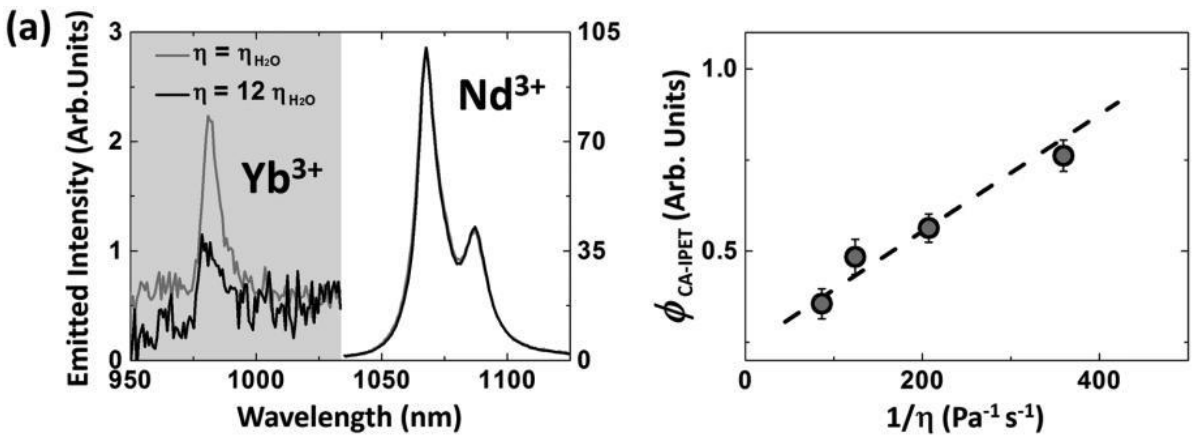

Figure 2. Dependence of IPET efficiency on viscosity. a) Emission spectra of the mixed LNF under optical excitation at $808 \mathrm{~nm}$ (i.e., optical excitation of doped SLNP:Nd) as obtained for two different viscosities of the solvent. b) Dependence of the CA-IPET efficiency on the inverse of fluid viscosity $\eta$. Symbols are experimental data and dashed line is a guide for the eyes.

SLNPs caused by, e.g., particle agglomeration or temperatureinduced Ostwald ripening. Figure $3 b$ shows SLNPs average size from TEM experiments conducted on the mixed LNF at different temperatures (see Figure S3 in the Supporting Information), confirming that within the error bars the size of the SLNPs remains unchanged. The two trends observed in the $\varphi$ CA-IPET versus temperature experiment may reveal the existence of a crossover in the molecular structure of water at $\mathrm{H} 45{ }^{\circ} \mathrm{C}$, which would modify the dynamics of interparticle

collisions and change the dependence of $\varphi$ CA-IPET on temperature. Note also that the molecular structure of water at temperatures below $45{ }^{\circ} \mathrm{C}$ minimizes the interaction between SLNPs, which makes $\varphi$ CA-IPET weakly dependent on temperature in this range. As mentioned above, the existence of this $45^{\circ} \mathrm{C}$ water crossover was reported in several studies of the optical, mechanical, and dielectric properties of water. ${ }^{[7]} \mathrm{We}$

\section{(a)}

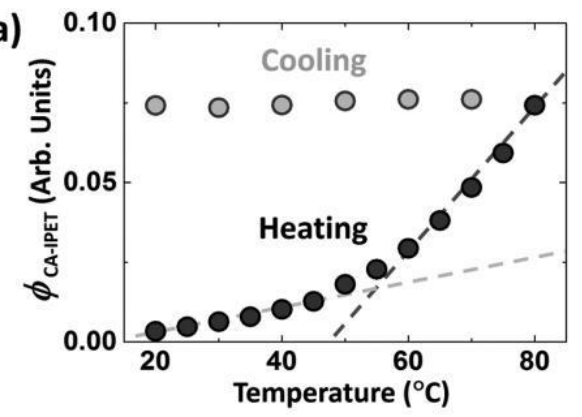

(b)

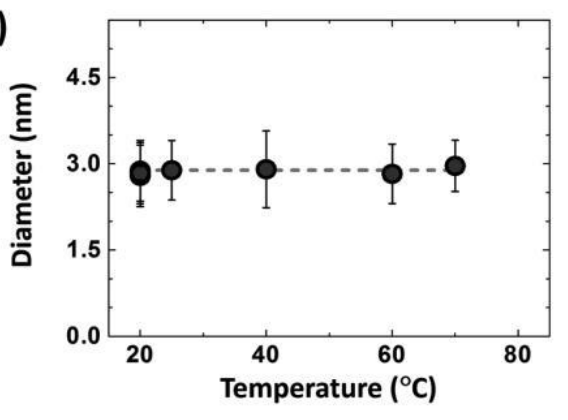

will relate this anomaly to the temperature-induced dissocia-tion of water molecules clusters later in this report.

Figure $3 \mathrm{a}$ shows that although $\varphi_{\mathrm{CA}}$-IPET increases by more than one order of magnitude when subjected to a heating

cycle, it remains temperature independent when cooled. This nonreversibility is not due to a CA-IPET process, in which the SLNPs involved in the collision events should not be altered. Clearly, the collision events permanently modify the SLNPs somehow. Figure $3 \mathrm{~b}$ shows TEM results that rule out structural and morphological changes in the nanoparticles after collision events. Therefore the observed spectral hys-teresis may be due to interparticle ion exchange during col-lision events. Figure $3 \mathrm{c}$ shows a schematic representation of the ion-exchange mechanism. When an SLNP:Nd and an SLNP:Yb collide, during the time interval of physical contact the SLNPs exchange superficial atoms, $\mathrm{Nd}^{3+}$ and $\mathrm{Yb}^{3+}$ ions

(c)
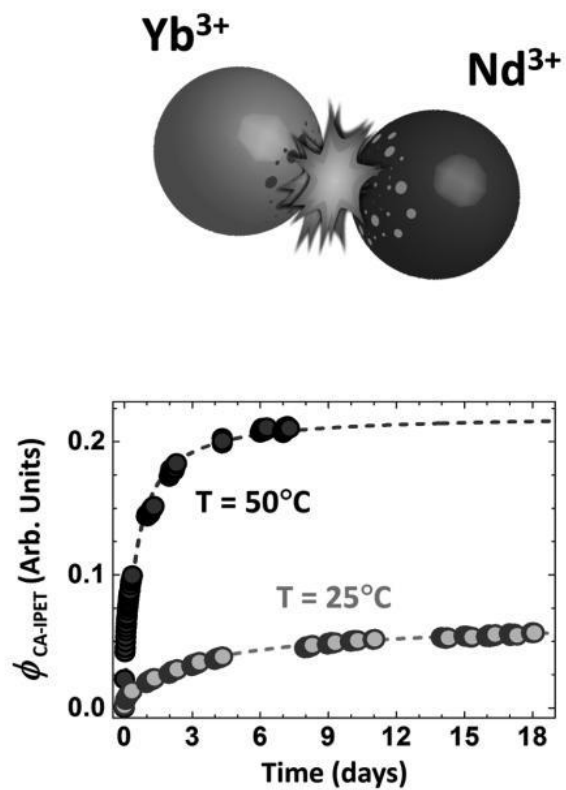

(d)

Figure 3. Dependence of IPET efficiency on temperature. a) Temperature dependence of the CA-IPET efficiency as obtained during a heating (black) and cooling (gray) cycle. Dashed lines are guides to the eyes in order to make evident the existence of two distinguished regimes. b) Diameter of the SLNPs estimated from TEM images of mixed LNF heated at diverse temperatures. c) Schematic representation of a collision-assisted interparticle ion exchange process. d) Long-term evolution of CA-IPET efficiency as obtained at two different temperatures $\left(25^{\circ} \mathrm{C}\right.$ (gray) and $50^{\circ} \mathrm{C}$ (black)). Symbols are experimental data and dashed lines are guides to the eyes. 
among them, and a fraction of $\mathrm{Yb}^{3+}\left(\mathrm{Nd}^{3+}\right)$ ions are incorporated on the surface of the SLNP:Nd (SLNP:Yb). After this collision-assisted ion exchange occurs, the distance between $\mathrm{Nd}^{3+}$ (donor) and $\mathrm{Yb}^{3+}$ (acceptor) ions is on the order of the SLNP size $(2.9 \mathrm{~nm})$, much lower than the average distance between SLNPs in the LNF $(\approx 10 \mathrm{~nm})$, which was the distance between ions initially. Due to the fact that the distance between donor and acceptor ions is significantly reduced after the ion exchange, $\varphi$ CA-IPET increases. This explains the maximum value of $\varphi$ CA-IPET experimentally obtained after the heating and cooling cycle. In addition, once ion exchange has occurred, no further collisions are needed for the effi-

cient energy transfer. Thus the lower collision rate (the lower

temperature) during the cooling procedure does not lower

$$
\varphi_{\text {CA-IPET }}
$$

To experimentally verify the presence of interparticle ion exchange in the LNFs, we keep their temperature constant and measure the long-term evolution of $\varphi$ CA-IPET. Note that at any given temperature collisions between SLNPs happen at a given rate that is proportional to this temperature. Thus ion exchange is occurring, and the $\varphi$ CA-IPET increases with time until it reaches saturation. The time needed to reach saturation is strongly temperature dependent. Higher temperatures increase collision rates, speed up the ion exchange process, and shorten the time to saturation. Figure $3 \mathrm{~d}$ shows the long-term evolution of $\varphi$ CA-IPET at 25 and $50{ }^{\circ} \mathrm{C}$ for a mixed LNF. As predicted for the CA-IPET process in the presence of ion exchange, experimentally we find saturation dynamics at both temperatures and a shorter saturation time at the higher temperature. Figure $3 d$ and Figure S9 (Supporting Information) also reveal that a more efficient inter-

particle ion exchange is produced at higher temperatures, as higher $\varphi$ CA-IPET values, together with a corresponding important shortening of the lifetime of the donor ions, are observed after long-term evolution at $50{ }^{\circ} \mathrm{C}$. X-ray diffraction measurements and TEM images of the LNF after these experiments (Figures S2 and S4 and Table S1, Supporting Information) confirm that the LNP has not suffered significant morphological modifications during long-term evolution. Figure $3 \mathrm{~d}$ shows experimental data indicating that the changes in the luminescent properties of the mixed LNF can only be understood in terms of a synergy between interparticle collisions and ion exchange events. Note that we assume that ion exchange occurs in a colloidal suspension of LNPs. This has also been reported by several researchers, although they did not correlate it with interparticle collisions. ${ }^{[12]}$ The effect of the long-term evolution at diverse temperatures (i.e., ion exchange saturation in the two regimes of water) on the temperature dependence of the $\varphi$ CA-IPET is studied in Section S5 (Supporting Information).

Figures 2 and 3 show experimental data that we now analyze using a simple model in order to correlate the temperature dependence of $\varphi$ CA-IPET with a crossover in the molecular behavior of water at $\approx 45^{\circ} \mathrm{C}$. For short time periods and far from saturation, the transfer efficiency ( $\varphi_{\mathrm{CA}}$-IPET) at a given temperature $\left(\mathrm{T}_{i}\right)$ is:

$\varphi$ CA-IPET $\left(T_{i}\right)^{\prime}=\varphi$ CA-IPET $\left.\left(T_{i-1}\right)\right)^{\prime}+\varphi\left(T_{\mathrm{i}}\right) \Delta t$ where $t$ is the measurement lap time at each $T$ (where $T_{i}{ }^{-} 1$ is the previous measured temperature), and $\varphi$ is the total number of successful collisions between LNPs (i.e., collisions leading to ion exchange) per unit time. The successful collisions are quantified by multiplying the total number of collisions per unit time by the probability of obtaining ion transfer $(K)$ and the probability that there is energy transfer between ions $(W)$. Because the total number of collisions per unit time is proportional to the temperature-dependent diffusion constant $(D)$, the proportionality expression for $K$ is:

$$
\kappa\left(T_{i}\right) \propto \frac{\phi \text { CA-IPET }\left(T_{i}\right)-\phi \text { CA-IPET }\left(T_{i-1}\right)}{D\left(T_{i}\right) W\left(T_{i}\right)}
$$

The diffusion constant is given by $D=k_{\mathrm{B}} T /(3 \pi \eta D \mathrm{NP})$, where $k_{\mathrm{B}}$ is the Boltzmann constant. Knowing the temperature dependence of $W$ and $\eta$ (see the data in Section S6 in the Supporting Information), we can determine the temperature dependence of $\kappa$ from the experimental data shown in Figure 3a. Figure 4a shows the temperature dependence of $K$, which remains approximately constant below $45^{\circ} \mathrm{C}$ and is thermally activated at higher temperatures, growing according to logarithmic dependence on the inverse of the absolute temperature. The low $\varphi$ CA-IPET values below $45^{\circ} \mathrm{C}$ are caused by the formation of water molecule cages around the SLNPs that fluctuate in time, impede collisions, and inhibit ion exchange. These cages disappear when temperature increases and therefore the collision-assisted ion exchange is thermally activated. ${ }^{[7 \mathrm{~d}\rfloor}$ The insets in Figure $4 \mathrm{~d}$ schematically show both situations.

The temperature-induced dissociation of water cages around the SLNPs indicates that, as previously proposed, ${ }^{[/]}$ there is a crossover between the regular water state and a hydrogen-bonded state. We now further explore the existence of this crossover by examining the dielectric behavior of liquid water to quantify at each temperature the value of i) the dipole moment and ii) the associated critical temperature $\left(T_{\mathrm{c}}\right)$ in terms of Curie-Weiss behavior. ${ }^{[13]}$ Figure $4 \mathrm{~b}, \mathrm{c}$ shows the results for (i) and (ii), respectively. Note how the dipole moment value falls between the accepted gas and ice values for the water molecule (1.84D and 2.6D, respectively), and how liquid water is always in the paraelectric phase. Since the value of the dipole moment of the water molecule is dependent upon the average number of clustered water molecules, ${ }^{[14]}$ the average number of water molecules in each cluster at a given temperature (i.e., the number of water molecules able to interact between them to form cages) shown in Figure $4 \mathrm{~d}$ is determined from the temperature dependence of the dipole moment shown in Figure 4b. Note that, for temperatures above $\approx 45^{\circ} \mathrm{C}$, the existence of pairs of hydrogenbonded water molecules starts to be unlikely, and this implies a decrease in the stability of the hydrogen bonded clusters surrounding the LNPs. This explains the disappearance of the

rates, and the temperature dependence of $\varphi$ CA-IPET shown in

Figure 3a. 

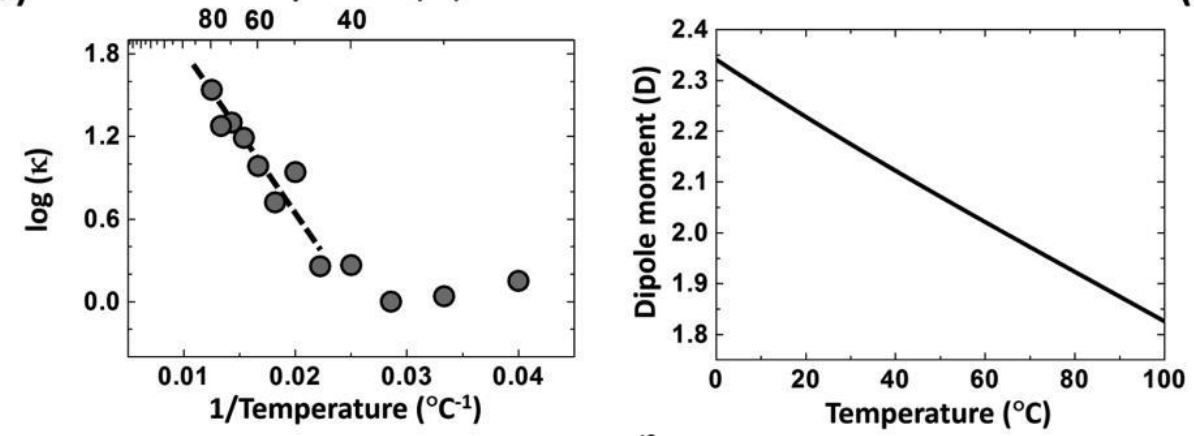

(c)
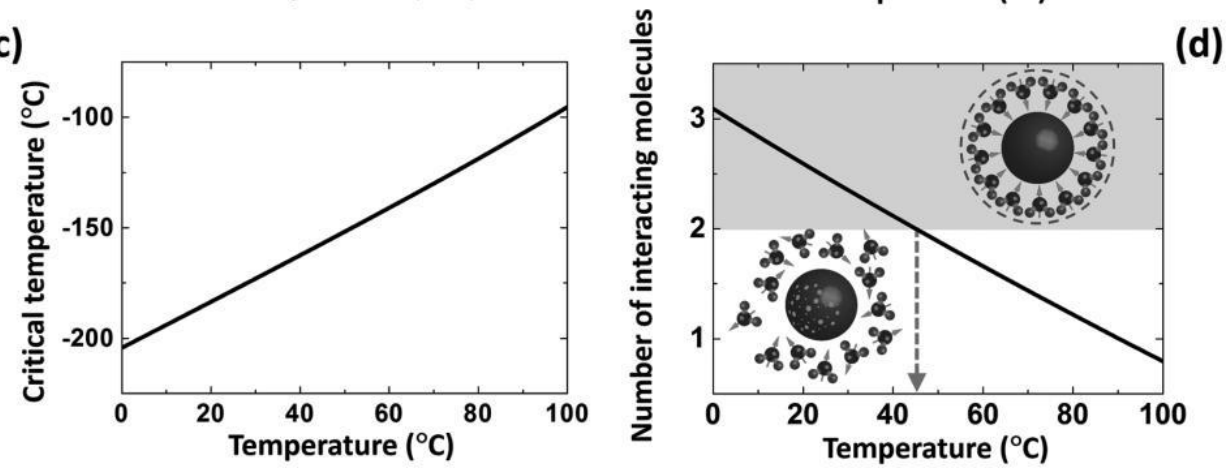

Figure 4. Crossover between water molecular behaviors. a) Logarithm of the ion transfer probability (except for a temperature independent constant) as a function of the inverse of temperature. A $1 / T$ linear dependence is only experimentally found for temperatures above $45{ }^{\circ} \mathrm{C}$ (dashed line). b) Dipole moment of water and c) critical temperature $T_{\mathrm{C}}$ (also known as Curie temperature) estimated from dielectric data as a function of temperature. d) Number of interacting molecules in water as a function of temperature. Insets: Schematic diagram of water molecules (top) interacting between them and so forming a cage that inhibits collisions and (bottom) being unable to interact among them and so allowing for interparticle collisions. Dashed arrow points out the crossover temperature.

\section{Conclusion}

In summary, we have used small luminescent nanoparticles doped with different rare earth ions as colloidal donor and acceptor units to study the interparticle energy transfer pro-cess in water-based nanofluids. We have found that there is interparticle energy transfer between colloidal small lumi-nescent nanoparticles. This process is mediated by inter-particle collisions, which increase the rate of nonreversible exchange of the luminescent ions between nanoparticles. The experimental data show that the interparticle energy transfer efficiency displays an unusual temperature depend-ence: A weak trend is observed below $45{ }^{\circ} \mathrm{C}$ and strong one above this temperature. This unusual temperature-dependent behavior indicates that there is a change in the molecular behavior of water at this temperature that is due to the exist-ence of a diffuse crossover at $\mathrm{H} 45{ }^{\circ} \mathrm{C}$ between a hydrogen-bonded and a regular state of water.

This indicates that the molecular structure of the solvent plays a decisive role in the interparticle dynamics of nano-fluids. In addition, the optical spectroscopy of colloidal small luminescent nanoparticles is a powerful tool in the study of molecular interactions of liquids. Therefore, the results pre-sented in this work open new avenues in which small lumi-nescent nanoparticles can be used as local molecular probes to study the molecular interactions of not just water but also a variety of other liquids.

\section{Experimental Section}

Reagents and Synthesis: Ammonium metavanadate $\left(\mathrm{NH}_{4} \mathrm{VO}_{3}\right.$; min. 99.0\%, Alfa Aesar), trisodium citrate dihydrate ( $\mathrm{Na}_{3} \mathrm{C}_{6} \mathrm{H}_{5} \mathrm{O} 7 ; 99+\%$, Sigma-Aldrich), gadolinium(III) nitrate hexahydrate $\left(\mathrm{Gd}\left(\mathrm{NO}_{3}\right) 3 \times 6 \mathrm{H}_{2} \mathrm{O}\right.$; 99.9\%, Alfa Aesar), neodymium(III) nitrate hex-ahydrate ( $\mathrm{Nd}\left(\mathrm{NO}_{3}\right) 3 \times$ $6 \mathrm{H}_{2} \mathrm{O} ; 99.9 \%$, Alfa Aesar), and ytterbium(III) nitrate pentahydrate $\left(\mathrm{Yb}\left(\mathrm{NO}_{3}\right)_{3} \times 5 \mathrm{H}_{2} \mathrm{O} ; 99.9 \%\right.$, Alfa Aesar) were used without further purification. Milli-Q deionized water (electrical resistivity $=18.2 \mathrm{M} \Omega$ $\mathrm{cm}^{-1}$ ) was utilized as a solvent. Spectra/Por 3 dialysis membrane, Standard RC Tubing, MWCO: 3.5 (Spectrum Laboratories, Inc.) was used for the dialysis of prepared colloidal suspensions. Colloidal suspensions of $\mathrm{Nd}^{3+}$ and $\mathrm{Yb}^{3+}$ doped GdVO4 SLNPs were prepared by following the procedure which is described in a recent paper. ${ }^{[15]} \mathrm{A}$ detailed description of the procedure is included in Section S1 (Supporting Information).

General Conditions for Structural Characterization: Samples for structural characterization were obtained by evaporation of aqueous colloidal solutions. X-ray diffraction measurements were performed with Rigaku SmartLab diffractometer using CuKa1.2 radiation $(\Lambda=0.15405 \mathrm{~nm})$. Diffraction data were recorded with a step of $0.01^{\circ}$ and a counting time of $1^{\circ} \mathrm{min}^{-1}$ over the $2 \theta$ range from $10^{\circ}$ to $100^{\circ}$. The study of particle morphology was accomplished using transmission electron microscope Tecnai G2 F20 (FEI) operated at $200 \mathrm{kV}$. The samples for TEM studies were placed on an ultrathin carbon film (3 nm) mounted on top of a supporting carbon holey film S187-4 (agar Scientific). 
General Conditions for Optical Characterization: Emission measurements were performed under excitation at $808 \mathrm{~nm}$ by means of a single-mode fiber-coupled laser diode. Laser light was collimated using a fiber port and focused through a $20 \times \mathrm{NA}=0.4$ microscope objective, that at the same time collects the lumines-cence of the NPs. Emission and excitation light were discriminated by means of a wavelength selective mirror and a $830-\mathrm{nm}$ long-pass filter. Finally, the luminescence of the LNPs was analyzed by a spec-trometer (Andor, Oxford Instruments) and recorded by an InGaAs detector. The temperature of the LNFs was controlled by employing a heating plate with an uncertainty of $0.1{ }^{\circ} \mathrm{C}$. The temperature was increased/decreased at a maximum rate of $2.5^{\circ} \mathrm{C} \mathrm{min}{ }^{-1}$. In order to guarantee the homogeneous heat distribution in the cuvette containing the sample, a thermal stabilization time of $15 \mathrm{~min}$ was hold before each measurement. A thermal gradient in the cuvette of less than $1{ }^{\circ} \mathrm{C}$ was achieved. The intensity decay curves of the luminescence of the donor ions in LNFs were obtained by exciting the LNFs at $808 \mathrm{~nm}$ using an optical parametric oscillator source (Spectra Physics). The light emitted by the LNFs was spectrally ana-lyzed by means of a spectrometer and then the intensity decay at

$892 \mathrm{~nm} .{ }^{4} \mathrm{~F}_{3 / 2} \rightarrow{ }^{4} \mathrm{lg}_{\mathrm{g} / 2}$ transition was measured by using a photomultiplier tube (R636-10, Hamamatsu) and a digital oscilloscope (LT372, LeCroy). Notice that this allows to measure the decay time of the ${ }^{4} \mathrm{~F}_{3 / 2}$, excited state which is also the one responsible for the 1060-nm emission.

General Procedure for Controlled Variation of Viscosity and Viscosity Estimation: Small controlled amounts of soap (Liquinox, Alconox) (with a viscosity of $\approx 500 \mathrm{mPa} \mathrm{s}$ ) were added to the LNF up to a volume $2.3 \%$ of the total volume of the LNF. The density of LNPs in the LNF was varied through this process by no more than a 2.3\%, which means that the average distance between LNPs was only increased up to $0.09 \mathrm{~nm}$. These variations are not significant in terms of spectral modifications.

\section{Acknowledgements}

This work was supported by the Spanish Ministerio de Educación y Ciencia (MAT2013-47395-C4-1-R and MAT2016-75362-C3-1-R) and by the COST Action CM1403. L.L.-P. thanks the Universidad Autónoma de Madrid for the "Formación de Personal Investi-gador (FPI-UAM)" program. P.H.-G. thanks the Spanish Ministerio de Economia y Competitividad (MINECO) for the Juan de la Cierva program. The authors from the University of Belgrade acknowledge the financial support of the Ministry of Education, Science and Technological Development of the Republic of Serbia (Project Nos. 45020 and 172056). M.I.M. thanks financial support from the
Spanish Ministerio de Economía y Competitividad (MICINN) Project FIS2015-69295-C3-3-P and the "María de Maeztu" Program Ref: MDM-2014-0377. The work of K.S. was supported by the Latvian National Research Program IMIS2 (Grant No. 302/2012).

[1] a) W. Yu, H. Xie, J. Nanomater. 2012, 2012, 1; b) O. Mahian, A. Kianifar, S. A. Kalogirou, I. Pop, S. Wongwises, Int. J. Heat Mass Transfer 2013, 57, 582; c) L. Zhang, Y. Jiang, Y. Ding, M. Povey, D. York, J. Nanopart. Res. 2007, 9, 479.

[2] C. D. Brites, X. Xie, M. L. Debasu, X. Qin, R. Chen, W. Huang, J.Rocha, X. Liu, L. D. Carlos, Nat. Nanotechnol. 2016, 11, 851.

[3] P. Rodríguez-Sevilla, H. Rodríguez-Rodríguez, M. Pedroni, A. Speghini, M. Bettinelli, J. G. Solé, D. Jaque, P. HaroGonzález, Nano Lett. 2015, 15, 5068.

[4] J. B. Miller, N. Dandu, K. A. Velizhanin, R. J. Anthony, U. R. Kortshagen, D. M. Kroll, S. Kilina, E. K. Hobbie, ACS Nano 2015, 9, 9772.

[5] A. Bednarkiewicz, M. Nyk, M. Samoc, W. Strek, J. Phys. Chem. C 2010, 114, 17535.

[6] a) P. Gallo, K. Amann-Winkel, C. A. Angell, M. A. Anisimov, F. D. R. Caupin, C. Chakravarty, E. Lascaris, T. Loerting, A. Z. Panagiotopoulos, J. Russo, Chem. Rev. 2016, 116, 7463; b) A. Nilsson, L. G. Pettersson, Nat. Commun. 2015, 6, 8998.

[7] a) L. Maestro, M. Marqués, E. Camarillo, D. Jaque, J. G. Solé, J. Gonzalo, F. Jaque, J. C. D. Valle, F. Mallamace, H. Stanley, Int. J. Nanotechnol. 2016, 13, 667; b) J. C. del Valle, E. Camarillo, L. Martinez Maestro, J. A. Gonzalo, C. Aragó, M. Marqués, D. Jaque, G. Lifante, J. G. Solé, K. Santacruz-Gómez, Philos. Mag. 2015, 95, 683; c) F. Mallamace, C. Corsaro, H. E. Stanley, Sci. Rep. 2012, 2, 993; d) J. G. Davis, K. P. Gierszal, P. Wang, D. Ben-Amotz, Nature 2012, 491, 582.

[8] a) J. Shi, P. W. Kantoff, R. Wooster, O. C. Farokhzad, Nat. Rev. Cancer 2017, 17, 20; b) D. Jaque, L. M. Maestro, B. Del Rosal, P.Haro-Gonzalez, A. Benayas, J. Plaza, E. M. Rodriguez, J. G. Sole, Nanoscale 2014, 6, 9494.

[9] a) F. Wang, R. Deng, J. Wang, Q. Wang, Y. Han, H. Zhu, X. Chen, X. Liu, Nat. Mater. 2011, 10, 968; b) S. Sarkar, B. Meesaragandla, C. Hazra, V. Mahalingam, Adv. Mater. 2013, 25, 856.

[10] S. Hao, G. Chen, C. Yang, Theranostics 2013, 3, 331.

[11] D. Jaque, M. Ramirez, L. Bausa, J. G. Solé, E. Cavalli, A. Speghini, M. Bettinelli, Phys. Rev. B 2003, 68, 035118.

[12] a) S. Han, X. Qin, Z. An, Y. Zhu, L. Liang, Y. Han, W. Huang, X. Liu, Nat. Commun. 2016, 7, 13059; b) M. Deng, L. Wang, Nano Res. 2014, 7, 782; c) C. Dong, F. C. van Veggel, ACS Nano 2008, 3, 123.

[13] C. Malmberg, A. Maryott, J. Res. Natl. Bur. Stand. 1956, 56, 1.

[14] J. Gregory, D. Clary, K. Liu, M. Brown, R. Saykally, Science 1997, 275, 814.

[15] B. del Rosal, A. Pérez-Delgado, M. Misiak, A. Bednarkiewicz, A. S. Vanetsev, Y. Orlovskii, D. J. Jovanovic', M. D. Dramic'anin, U. Rocha, K. U. Kumar, J. Appl. Phys. 2015, 118, 143104. 